\title{
Role of autophagy in drug resistance and regulation of osteosarcoma (Review)
}

\author{
YANCHUAN PU, JIN WANG and SHIZHONG WANG \\ Department of Orthopedics, Wuwei People's Hospital, Wuwei, Gansu 733000, P.R. China
}

Received December 2, 2020; Accepted November 17, 2021

DOI: $10.3892 / \mathrm{mco} .2022 .2505$

\begin{abstract}
Osteosarcoma (OS) is a malignant tumor originating from primitive mesenchymal tissue that occurs mostly in children and adolescents. It is the most common type of malignant tumor originating from bone. The combination of chemotherapy and surgery is an important treatment strategy for OS; however, multidrug resistance frequently leads to failure of chemotherapy for OS. Autophagy is considered an important mechanism through which bone tumor cells escape apoptosis; inhibition of autophagy may significantly increase the sensitivity of tumor cells to chemotherapeutic drugs. The present review discusses the relationship between chemotherapy resistance and autophagy-related genes, the regulation of autophagy in OS, as well as drugs that inhibit protective autophagy in tumors or cause autophagic death of OS cells or increase their sensitivity to chemotherapy drugs, thereby reducing chemotherapy resistance and increasing efficacy.
\end{abstract}

\section{Contents}

1. Introduction

2. Autophagy and chemotherapy resistance

3. Regulation of autophagy in OS

4. Drugs affecting autophagy and chemotherapy efficacy for OS

5. Summary

\section{Introduction}

Osteosarcoma (OS), a malignant tumor type that originates in mesenchymal tissue, is most common in children and adolescents. OS occurs in the metaphysis of long bones with a rich blood supply. It is highly malignant and characterized by early metastasis, rapid disease progression, a high mortality

Correspondence to: Professor Shizhong Wang, Department of Orthopedics, Wuwei People's Hospital, 24 Xuanwu Street, Wuwei, Gansu 733000, P.R. China

E-mail: wangsz0325@163.com

Key words: osteosarcoma, autophagy, drug resistance, drug regulation rate and frequent clinical treatment failure $(1,2)$. At present, OS is primarily treated by a combination of neoadjuvant chemotherapy, surgery and postoperative chemotherapy. Surgical treatment alone is ineffective and associated with frequent recurrence and lung metastasis; the 5-year survival rate of patients is only $15-20 \%$ (3). The combination of chemotherapy and surgery is an important treatment strategy for OS; however, multidrug resistance frequently leads to failure of chemotherapy for OS. The causes of drug resistance of OS cells are diverse and include low drug absorption, evasion of apoptosis, abnormal function of microRNAs (miRNAs), autophagy, altered membrane permeability and the DNA damage response. Changes in autophagy are thought to cause drug resistance in OS and the proliferation of malignant cells may result in treatment failure.

In autophagy, cells create autophagolysosomes from lysosomes to degrade damaged organelles, such as mitochondria and macromolecules. Autophagy has an important regulatory role in cell growth, development, differentiation and death (4-7). According to certain researchers, autophagic death may be an important way to eliminate tumor cells resistant to apoptosis due to gene mutations (8). The primary mechanism of action of chemotherapy drugs is the induction of tumor cell apoptosis; however, chemotherapeutics may also induce autophagy of tumor cells. Apoptosis and autophagy are related but independent processes (9). Regulating the autophagy of OS cells to reduce their resistance to chemotherapeutic drugs is an important consideration in the development of novel treatment strategies for OS.

\section{Autophagy and chemotherapy resistance}

\section{Basic concepts}

Autophagy is an evolutionarily conserved process that involves lysosomal enzymatic degradation of damaged organelles and proteins to maintain cellular homeostasis. Under stress conditions and during cell death, high levels of autophagy are induced; thus, it is thought that autophagy may initiate cell death, although this has been controversial. Autophagy is complex, involving various signaling pathways that promote cell death (10). Cells may undergo autophagy due to a lack of nutrients, blood oxygen and growth factors, and due to cellular toxicity caused by proteins or organelles, and disturbances in their internal environment (10). Autophagy is classified into macroautophagy, microautophagy and 
chaperone-mediated autophagy. The different types of autophagy are presented in Fig. 1.

Chemotherapy, surgery and radiotherapy are the three major treatments for cancer. Chemotherapy involves the use of drugs to kill cancer cells and is currently one of the most effective treatments. Chemotherapeutics, as systemic treatments, diffuse throughout the body via the blood circulation. As such, for tumors that have a tendency to spread throughout the body, as well as for advanced tumors that have undergone metastasis, chemotherapy is the primary treatment (11).

\section{Role of autophagy in inhibiting tumor progression}

DNA and cell organelles may be damaged by physical, chemical and biological carcinogens, such as radiation, aflatoxin and viruses, respectively, which disrupt cellular metabolism. The accumulation of metabolic waste and a lack of cellular energy activates autophagy-related genes (ATGs), which initiate autophagy. Autophagy may eliminate damaged organelles, degrade self-folded proteins and maintain genomic stability, thereby inhibiting tumor development. It has been indicated that, in early tumor cells, autophagy is either present at low levels or absent. Loss of autophagy may cause accumulation of mutations, leading to tumor formation and metastasis. Therefore, promoting autophagy may inhibit tumor formation and development (12). Mele et al (13) determined that curcumin may increase Beclin-1 and microtubule-associated protein 1 light chain $3 \beta$ (LC3B) protein levels and inhibit AKT-mammalian target of rapamycin (mTOR) and other pathways, thereby inducing autophagy.

During tumor development, tumor cells proliferate rapidly; this process requires high levels of nutrients. However, rapid growth is not possible when there are insufficient blood vessels in the tumor tissue. When the energy demands of solid tumor cells are not met due to a lack of oxygen and nutrients, Liver kinase B1 is activated to phosphorylate adenosine monophosphate-activated kinase (AMPK). Activated AMPK phosphorylates Ser863, while TSC2 is able to activate Ras homolog, mTORC1 binding to inhibit mTORC1 and induce autophagy (14). When in a hypoxic state, tumor cells obtain energy from anaerobic glycolysis, which generates high levels of reactive oxygen species that further promote autophagy (15). During tumor development, cells are able to resist various environmental stressors by inducing autophagy. Autophagy may degrade damaged organelles and misfolded proteins, provide energy for tumor cells and promote the spread of tumor cells. Furthermore, tumor cell death induced by autophagy may lead to moderate inflammation; this promotes new blood vessels that may dilate into the tumor tissue to provide nutrition supporting tumor growth. When environmental stress is severe, tumor cells may enter into a state of reversible dormancy due to autophagy and exist in the host for a long period of time (16). Therefore, moderate levels of autophagy are conducive to the survival and development of tumor cells in vivo.

\section{Autophagy and chemotherapy tolerance}

It is thought that autophagy has an important role in the occurrence, development and treatment of tumors. Although chemotherapy has a significant beneficial effect in numerous patients, acquired drug resistance has become a major reason for treatment failure. Numerous studies have indicated that a variety of chemotherapeutic drugs may induce autophagy $(10,17)$. Furthermore, there is a correlation between autophagy and tumor chemoresistance.

Chemotherapy induces apoptosis of cancer cells; however, cancer cells frequently protect themselves by inducing autophagy, thereby avoiding apoptosis, which markedly reduces the efficacy of chemotherapy. Liu et al (18) used MTT and Hoechst 33342 staining, as well as flow cytometry, to detect apoptosis of A549 lung cancer cells after chemotherapy; they also used the autophagy inhibitor 3-methyladenine (3-MA) to study the relationship between autophagy and apoptosis in cancer cells. Their experiments indicated that cisplatin (DDP) and paclitaxel may induce autophagy and apoptosis of A549 lung cancer cells. Studies have also indicated that salivary gland adenoid cystic cancer cells caused by autophagy are resistant to DDP, which frequently leads to chemotherapy failure (19). Transmission electron microscopy is able to detect the expression of the autophagy marker LC3 and trace amounts of p62 also indicate autophagy induced by DDP. Furthermore, downregulation of Beclin-1 via 3-MA or RNA interference may enhance DDP-induced apoptosis. Therefore, the induction of protective autophagy by chemotherapy enhances the chemotherapeutic resistance of tumor cells.

\section{Regulation of autophagy in OS}

\section{mTOR}

mTOR has important roles in cell growth and metabolism, as well as in the regulation of autophagy. mTOR is an atypical serine/threonine protein kinase that is able to control intracellular mRNA translation and protein synthesis. Changes in mTOR signaling are common in numerous tumor types, including OS. Kim et al (20) indicated that when the body is in a normal nutritional state, the PI3K/AKT/mTOR pathway is activated to inhibit autophagy and cells proliferate normally. However, when cells are underfed or stressed, mTOR is inhibited and autophagy thus activated, causing uncontrolled cell growth and proliferation, as well as inhibition of apoptosis, eventually leading to tumor progression and metastasis (21).

\section{High mobility group protein 1 (HMGB1)}

HMGB1 is associated with damage to chromatin and is also involved in the construction and stabilization of nucleosomes and DNA damage repair. Furthermore, HMGB1 is an important regulator of autophagy in chemoresistant OS $(22,23)$. Zhang et al (24) indicated that HMGB1 competitively binds Beclin-1 during autophagy and regulates autophagy by controlling the dissociation of the Beclin1/Bcl-2 complex. HMGB1 also binds cell surface receptors, activating downstream signaling pathways to stimulate cell proliferation and migration, as well as autophagy. In short, the high expression of HMGB1 observed in OS tissue is related to the occurrence and development of tumors. Downregulation of HMGB1 may hinder tumor cell metastasis (23); thus, HMGB1 has an important regulatory role in tumor progression.

\section{miRNAs}

miRNAs are highly conserved non-coding RNAs ( 22 oligonucleotides) that repress gene expression by binding a target mRNA at the 3'-untranslated region, thus inhibiting translation or inducing degradation. miRNAs regulate cell differentiation 


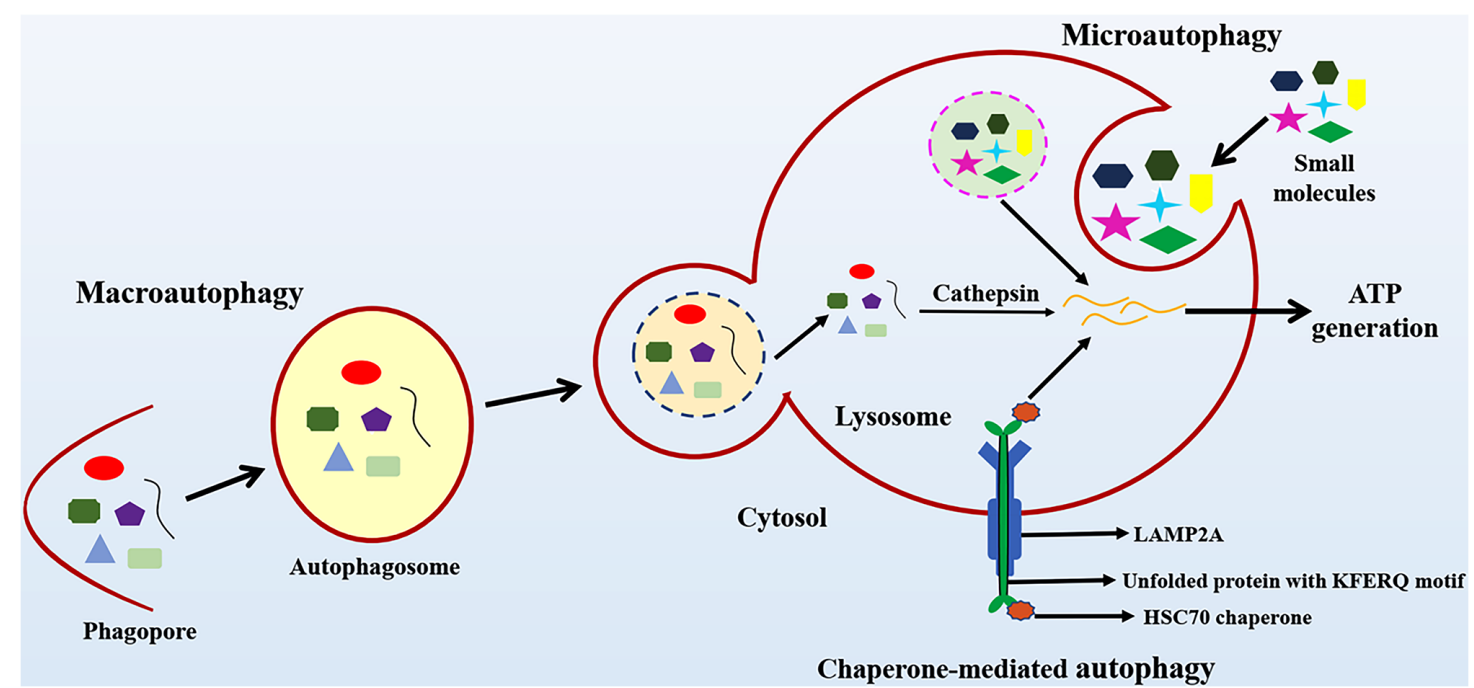

Figure 1. Schematic illustration of the different types of autophagy. Macroautophagy is a common autophagic pathway, mitophagy is a specific type of autophagy, which may occur in mitochondria, and chaperone-mediated autophagy is a selective type of autophagy with a unique mechanism. KFERQ is a pentapeptide motif. HSC70, heat shock cognate $71 \mathrm{kDa}$ protein; LAMP, lysosomal-associated membrane protein.

and development, the nervous system, immunity, viral infection, DNA repair, cell junctions, cell-to-cell communication, cellular reprogramming and metabolism $(25,26)$. Numerous studies have demonstrated the important role of miRNAs in the occurrence, regulation and progression of human OS. Certain miRNAs act as tumor suppressors, while others act as oncogenes (27). In recent years, the correlation between miRNAs and autophagy has attracted much attention. While most miRNAs are downregulated in OS cell lines, Mutlu et al (28) reported that, following suppression of autophagy-associated miRNAs by adriamycin and rapamycin, the expression of certain miRNAs, including miR-3141, miR-4296, miR-133b and miR-720, was markedly increased. Chen et al (29) and Niu et al (23) indicated that miRNAs have an important regulatory role in autophagy. Numerous miRNAs have also been reported to suppress the development of resistance and sensitivity to drugs by controlling and blocking autophagy. According to various studies $(23,29,30)$, miR-101 not only has a significant inhibitory effect on OS cell proliferation, but may also promote apoptosis, while reducing the expression of the autophagy-related proteins Beclin1 and LC3B; these results suggest that miR-101 affects the proliferation and apoptosis of OS cells by regulating the expression of autophagy genes. miR-22, which has an important role in the regulation of autophagy, functions as both a tumor suppressor gene and proto-oncogene. It has a key role in cell growth, proliferation, migration, invasion and aging (31). Wang et al (32) and Li et al (33) indicated that miR-22 regulates HMGB1-induced autophagy and has an important role in the proliferation and migration of OS. Overexpression of miR-22 inhibits cell proliferation and the formation of OS cell colonies in patients treated with anti-tumor drugs, suggesting that miR-22 has potential for reducing the development of drug resistance during OS chemotherapy.

\section{p53}

p53 regulates the cell cycle and apoptosis and affects the efficacy of chemotherapeutic drugs (34). As an important tumor suppressor gene, p53 is involved in the regulation of autophagy. Cytoplasmic p53 inhibits autophagy. Pitolli et al (35) determined that nutritional deficiencies, changes in the cellular environment and DNA damage activate p53 and AMPK pathways in the nucleus, resulting in the phosphorylation of tuberous sclerosis complex, inhibition of mTOR activity and the induction of autophagy. p53 is also able to activate pro-apoptotic proteins to dissociate the Beclin1-Bcl-2 complex, thereby promoting autophagy.

\section{Beclin-1}

The most common mechanism of autophagy induction in patients with OS is the activation of Beclin-1 via upstream mediators (30). Beclin-1 was the first mammalian-related autophagy regulatory gene to be identified. Beclin-1 dysfunction may lead to immune dysfunction and tumorigenesis. Zhang et al (36) reported that chemotherapeutic drugs, such as DDP, doxorubicin and methotrexate, induce upregulation of Beclin-1 expression in OS cells, while knocking down the Beclin-1 gene inhibited OS cell proliferation, metastasis and invasion. OS cells are more sensitive to chemotherapy when the Beclin-1 gene is knocked down or an autophagy inhibitor is used (37). Beclin-1 has an important role in OS cell proliferation and tumor progression, and inhibition of autophagy may improve the efficacy of chemotherapy.

Atg- $4 B$

There are two major autophagy pathways in OS: The mTOR and class III phosphatidylinositol kinase (Ptdins3K) pathways (38). mTOR stimulates Ptdins3k activity and inhibits the formation of the mammalian orthologs of yeast Atg1 (ULK1/2) complex. The mTOR inhibitor rapamycin induces autophagy-mediated cell death in gliomas. Ptdins3K synthesizes PI3K, which provides a binding site for ATGs during the formation of autophagosomes. These two pathways regulate the formation of $\mathrm{LC} 3 \mathrm{~B}$ liposomes by regulating the activity of Atg4 and Atg7. Atg4B, which activates LC3B, catalyzes the cleavage of the carboxyl end of LC3B (39).

Shi et al (40) indicated that the anti-tumor effect of the drug NSC185058 is related to Atg4B function and inhibition 
of autophagy. A high concentration of NSC185058 reduces the viability of Saos-2 cells. Knocking down Atg4B leads to autophagic defects in Saos-2 OS cells. In addition, the use of Atg4B protein antagonists to reduce Saos-2 OS cell viability was linked to inhibition of autophagy; the antagonists had no effect on Atg4B-deficient OS cell lines. Thus, inhibition of autophagy is considered the primary mechanism underlying the anti-tumor activity of the drug NSC185058 (41).

\section{Drugs affecting autophagy and chemotherapy efficacy for OS}

Certain drugs promote autophagy, while others inhibit it. Autophagy-inhibiting drugs include chloroquine and 3-MA. Autophagy inducers increase autophagy and killing of OS cells, i.e., they both promote protective autophagy and cause autophagic death. Autophagy inhibitors also increase tumor cell death, indicating that the regulation of autophagy may increase sensitivity to chemotherapeutic drugs, particularly in the presence of particular gene mutations and in tumor cells resistant to apoptosis. Most of the available drugs enhance sensitivity to chemotherapeutics by inhibiting protective autophagy.

\section{Autophagy inducers}

Tripterygium wilfordii. Tripterygium wilfordii is a traditional Chinese medicine that exerts pharmacological effects, including immunosuppression. Recently, Tripterygium wilfordii has been reported to also exert anti-tumor effects; it kills leukemia, multiple myeloma, liver cancer, melanoma and breast cancer cells (42-45). Hou et al (46) indicated that Tripterygium wilfordii inhibits the proliferation of OS cells but is not toxic to normal cells. Tripterygium wilfordii has been demonstrated to induce apoptotic and autophagic death of OS cells, thus significantly inhibiting OS cell proliferation; these effects were partially ameliorated by autophagy inhibitors.

Arsenic trioxide. Arsenic trioxide $\left(\mathrm{As}_{2} \mathrm{O}_{3}\right)$ was first used in the treatment of acute promyelocytic leukemia. It exhibits short-term efficacy in the treatment of stage III OS (47). Hashmi and Nishihori (48) reported that $\mathrm{AS}_{2} \mathrm{O}_{3}$ promoted autophagic cell death in human OS (HOS), but as the level of autophagy increases, so too does the level of HOS apoptosis. $\mathrm{AS}_{2} \mathrm{O}_{3}$ may also increase the level of autophagy in multidrug-resistant cells, such as MG63 cells. However, as the $\mathrm{As}_{2} \mathrm{O}_{3}$ concentration increases, the level of cell autophagy decreases following an initial increase, eventually reaching the basal level. A marker protein of early apoptosis was identified at this stage. The effects of $\mathrm{As}_{2} \mathrm{O}_{3}$ vary among OS cells with different characteristics. In multidrug-resistant cells, such as MG63 cells, $\mathrm{As}_{2} \mathrm{O}_{3}$ induces protective autophagy, which partially alleviates cell death, while in chemotherapy-sensitive cells, such as $\mathrm{HOS}$ cells, $\mathrm{As}_{2} \mathrm{O}_{3}$ induces autophagic death. Zhang et al (49) indicated that $\mathrm{As}_{2} \mathrm{O}_{3}$ induced protective autophagy in gastric cancer cells and the addition of autophagy inhibitors markedly increased apoptosis.

Rapamycin. The immunosuppressive agent rapamycin is primarily used to prevent immune rejection after organ transplantation. Recently, rapamycin was reported to exert significant anti-tumor effects $(50,51)$. mTOR, an upstream regulator of autophagy, phosphorylates ULK1 and ULK2, thereby inhibiting autophagy (52). As an mTOR inhibitor, rapamycin inhibits the transition of the cell cycle from G0/G1 to $\mathrm{S}$, as well as protein transcription and translation, thereby inhibiting tumor cell proliferation (53). Rapamycin is also an effective autophagy inducer. Protective autophagy inhibits the death of certain tumor cells (54). Saraf et al (55) indicated that the autophagy inhibitor chloroquine, used in combination with rapamycin, inhibited protective autophagy in OS and increased rapamycin-mediated inhibition of tumor cell proliferation, ultimately increasing the sensitivity of chemotherapeutic drugs.

\section{Autophagy inhibitors}

Autophagy inhibitors have not yet been used on their own in the clinical setting due to a lack of specificity, i.e., due to their toxicity to normal cells. However, the efficacy of chemotherapeutic drugs is commonly enhanced by adding autophagy inhibitors to the regimen. Kocaturk et al (56) indicated that the level of autophagy in MG63 cells was significantly reduced by the autophagy inhibitor 3-MA; its use in combination with DDP significantly increased MG63 cell death. After U2OS cells had been pretreated with the autophagy inhibitor chloroquine, treatment with the Akt kinase inhibitor MK-2206 further inhibited their activity (57). Saraf et al (55) reported that treatment of OS cells with chloroquine and rapamycin inhibited protective autophagy, thereby inhibiting tumor cell proliferation and enhancing sensitivity to chemotherapeutic drugs. Further research on autophagy inhibitors is important as a means of increasing the sensitivity to, and thus the efficacy of, chemotherapeutic drugs.

\section{Survivin inhibitors}

Survivin, an inhibitor of apoptosis protein, regulates mitosis and apoptosis. Survivin has been detected in most types of tumor tissues and may increase tumor cell apoptosis and chemotherapy sensitivity (58); thus, survivin has been considered a target in OS treatment. YM155, a specific inhibitor of survivin, inhibits the proliferation of various tumor cell types and is considered safe and effective (59). Khan et al (60) indicated that YM155 inhibits proliferation, induces autophagy and apoptosis, and reduces the expression of survivin mRNA in F5M2 cells. Waligórska-Stachura et al (59) determined that survivin is highly expressed in OS cells and is related to the degree of malignancy. YM155 inhibited Saos-2 and MG63 cell proliferation and invasion and promoted apoptosis. It also increased sensitivity to the chemotherapeutic doxorubicin. Coumar et al (61) demonstrated that YM155 inhibited the proliferation of liver cancer cells and is involved in the induction of autophagic death of stem cancer cells. Church and Talbot (62) indicated that YM155 induced autophagy in the breast cancer cell line MDA-MB-231; this induction of autophagy also promoted apoptosis. The mechanism underlying the effect of YM155 on malignant tumors has emerged as an important research target. The relationship between YM155 and autophagy in OS remains to be further elucidated. In particular, it remains to be determined whether autophagy inducers are able to increase the efficacy of YM155.

\section{Photodynamic therapy $(P D T)$}

PDT involves intravenous or local injection of photosensitizers into the body. Selective aggregation of tumor cells occurs 
when using a particular wavelength of laser irradiation; this causes tumor cells to produce large quantities of cytotoxic singlet oxygen and oxygen free radicals, which may inhibit tumor cell proliferation and spread $(63,64)$. PDT damages organelles, which in turn induces apoptosis and autophagy. A certain level of autophagy may improve cell viability under stress. Furthermore, when apoptosis genes are mutated or suppressed, autophagic death becomes the primary mechanism of cell death. Aloe-emodin, an anti-tumor drug and photosensitizer, induces autophagy in MG63 cells, which leads to an early anti-apoptotic effect (65). The effects of the autophagy induced by PDT vary by cell type and dose; increasing the efficacy of PDT is an important goal of future research.

\section{Summary}

Autophagy has an important role in the onset, progression and treatment of OS. The relationship between autophagy and tumor behavior is complex; autophagy exerts different regulatory effects according to the stage of the tumor. At present, clinical treatments of OS are not ideal. High-dose adjuvant chemotherapy may induce protective autophagy and lead to drug resistance, and is accompanied by serious side effects. Chemotherapy resistance may markedly affect treatment outcomes. Autophagy-related factors such as mTOR, Beclin-1, miRNA, HMG family proteins and ATGs are involved in chemoresistance in OS. Modulating the autophagy pathway to reduce chemoresistance and increase tumor sensitivity to therapeutic drugs should improve the outcomes of OS. Although there are no clinical trials on osteosarcoma and autophagy, the combined application of autophagy inhibitors and chemotherapeutic drugs is receiving increased attention in the field of cancer treatment. Autophagy may reverse multidrug resistance, thereby increasing the sensitivity of tumor cells to drugs.

\section{Acknowledgements}

Not applicable.

\section{Funding}

No funding was received.

\section{Availability of data and materials}

Data sharing is not applicable to this article, as no datasets were generated or analyzed during the current study.

\section{Authors' contributions}

Conceptualization and methodology: SW; investigation: YP and JW; writing-original draft: YP; writing-review and editing: YP, JW and SW; visualization: SW; project administration: SW. All authors have read and approved the final manuscript. Data authentication is not applicable.

\section{Ethics approval and consent to participate}

Not applicable.

\section{Patient consent for publication}

Not applicable.

\section{Competing interests}

The authors declare that they have no competing interests.

\section{References}

1. Lu KH, Lu EW, Lin CW, Yang JS and Yang SF: New insights into molecular and cellular mechanisms of zoledronate in human osteosarcoma. Pharmacol Ther 214: 107611, 2020.

2. Otoukesh B, Abbasi M, Gorgani HO, Farahini H, Moghtadaei M, Boddouhi B, Kaghazian P, Hosseinzadeh S and Alaee A: MicroRNAs signatures, bioinformatics analysis of miRNAs, miRNA mimics and antagonists, and miRNA therapeutics in osteosarcoma. Cancer Cell Int 20: 254, 2020

3. Li Z, Xu D, Chen X, Li S, Chan MTV and Wu WKK: LINC01133: An emerging tumor-associated long non-coding RNA in tumor and osteosarcoma. Environ Sci Pollut Res Int 27: 32467-32473, 2020.

4. Mendes AC, Ciccone M, Gazolla B and Bahia D: Epithelial haven and autophagy breakout in gonococci infection. Front Cell Dev Biol 8: 439, 2020.

5. Levine B and Kroemer G: Autophagy in the pathogenesis of disease. Cell 132: 27-42, 2008.

6. Ma W, Wei S, Zhang B and Li W: Molecular mechanisms of cardiomyocyte death in drug-induced cardiotoxicity. Front Cell Dev Biol 8: 434, 2020.

7. Lin Y, Zhao WR, Shi WT, Zhang J, Zhang KY, Ding Q, Chen XL, Tang JY and Zhou ZY: Pharmacological activity, pharmacokinetics, and toxicity of timosaponin AIII, a natural product isolated from anemarrhena asphodeloides bunge: A review. Front Pharmacol 11: 764, 2020.

8. Liu W, Meng Y, Zong C, Zhang S and Wei L: Autophagy and tumorigenesis. Adv Exp Med Biol 1207: 275-299, 2020.

9. Blondy S, David V, Verdier M, Mathonnet M, Perraud A and Christou N: 5-Fluorouracil resistance mechanisms in colorectal cancer: From classical pathways to promising processes. Cancer Sci 111: 3142-3154, 2020.

10. Condello M, Mancini G and Meschini S: The exploitation of liposomes in the inhibition of autophagy to defeat drug resistance. Front Pharmacol 11: 787, 2020.

11. Whelan JS and Davis LE: Osteosarcoma, chondrosarcoma, and chordoma. J Clin Oncol 36: 188-193, 2018.

12. Lim J and Murthy A: Targeting autophagy to treat cancer: Challenges and opportunities. Front Pharmacol 11: 590344, 2020.

13. Mele L, Del Vecchio V, Liccardo D, Prisco C, Schwerdtfeger M, Robinson N, Desiderio V, Tirino V, Papaccio G and La Noce M: The role of autophagy in resistance to targeted therapies. Cancer Treat Rev 88: 102043, 2020.

14. Yang J, Ueharu $\mathrm{H}$ and Mishina Y: Energy metabolism: A newly emerging target of BMP signaling in bone homeostasis. Bone 138: 115467, 2020.

15. Tian Y, Song W, Xu D, Chen X, Li X and Zhao Y: Autophagy induced by ROS aggravates testis oxidative damage in diabetes via breaking the feedforward loop linking p62 and Nrf2. Oxid Med Cell Longev 2020: 7156579, 2020.

16. Kulka LAM, Fangmann PV, Panfilova D and Olzscha H: Impact of HDAC inhibitors on protein quality control systems: Consequences for precision medicine in malignant disease. Front Cell Dev Biol 8: 425, 2020.

17. Ashrafizadeh M, Tavakol S, Ahmadi Z, Roomiani S, Mohammadinejad R and Samarghandian S: Therapeutic effects of kaempferol affecting autophagy and endoplasmic reticulum stress. Phytother Res 34: 911-923, 2020.

18. Liu F, Liu D, Yang Y and Zhao S: Effect of autophagy inhibition on chemotherapy-induced apoptosis in A549 lung cancer cells. Oncol Lett 5: 1261-1265, 2013.

19. Tan Q, Liu Y, Deng X, Chen J, Tsai PJ, Chen PH, Ye M, Guo J and $\mathrm{Su} \mathrm{Z}$ : Autophagy: A promising process for the treatment of acetaminophen-induced liver injury. Arch Toxicol 94: 2925-2938, 2020.

20. Kim WK, Pyee Y, Chung HJ, Park HJ, Hong JY, Son KH and Lee SK: Antitumor activity of spicatoside A by modulation of autophagy and apoptosis in human colorectal cancer cells. J Nat Prod 79: 1097-1104, 2016. 
21. Yecies JL and Manning BD: mTOR links oncogenic signaling to tumor cell metabolism. J Mol Med (Berl) 89: 221-228, 2011.

22. Tang D, Loze MT, Zeh HJ and Kang R: The redox protein HMGB1 regulates cell death and survival in cancer treatment. Autophagy 6 : 1181-1183, 2010.

23. Niu J, Yan T, Guo W, Wang W and Zhao Z: Insight into the role of autophagy in osteosarcoma and its therapeutic implication. Front Oncol 9: 1232, 2019.

24. Zhang J, Kou YB, Zhu JS, Chen WX and Li S: Knockdown of HMGB1 inhibits growth and invasion of gastric cancer cells through the NF- $\mathrm{BB}$ pathway in vitro and in vivo. Int J Oncol 44 1268-1276, 2014.

25. Berindan-Neagoe I, Monroig Pdel C, Pasculli B and Calin GA: MicroRNAome genome: A treasure for cancer diagnosis and therapy. CA Cancer J Clin 64: 311-336, 2014.

26. Gulino R, Forte S, Parenti R, Memeo L and Gulisano M: MicroRNA and pediatric tumors: Future perspectives. Acta Histochem 117: 339-354, 2015

27. Llobat L and Gourbault O: Role of MicroRNAs in human osteosarcoma: Future perspectives. Biomedicines 9: 463, 2021.

28. Mutlu H, Mutlu S and Bostanciklıŏlu M: Profiling of autophagy-associated microRNAs in the osteosarcoma cell line of U2OS. Anticancer Agents Med Chem 21: 1732-1737, 2021.

29. Chen R, Wang G, Zheng Y,Hua Y and CaiZ: Drug resistance-related microRNAs in osteosarcoma: Translating basic evidence into therapeutic strategies. J Cell Mol Med 23: 2280-2292, 2019.

30. Jamali Z, Taheri-Anganeh M, Shabaninejad Z, Keshavarzi A, Taghizadeh H, Razavi ZS, Mottaghi R, Abolhassan M, Movahedpour A and Mirzaei $\mathrm{H}$ : Autophagy regulation by microRNAs: Novel insights into osteosarcoma therapy. IUBMB Life 72: 1306-1321, 2020.

31. Xia $\mathrm{H}$ and Hui KM: Mechanism of cancer drug resistance and the involvement of noncoding RNAs. Curr Med Chem 21: 3029-3041, 2014

32. Wang G, Shen N, Cheng L, Lin J and Li K: Downregulation of miR-22 acts as an unfavorable prognostic biomarker in osteosarcoma. Tumour Biol 36: 7891-7895, 2015.

33. Li X, Wang S, Chen Y, Liu G and Yang X: miR-22 targets the 3 UTR of HMGB1 and inhibits the HMGB1-associated autophagy in osteosarcoma cells during chemotherapy. Tumour Biol 35: 6021-6028, 2014.

34. Xu S, Gong Y, Yin Y, Xing $\mathrm{H}$ and Zhang N: The multiple function of long noncoding RNAs in osteosarcoma progression, drug resistance and prognosis. Biomed Pharmacother 127: 110141, 2020.

35. Pitolli C, Wang Y, Candi E, Shi Y, Melino G and Amelio I: p53-Mediated tumor suppression: DNA-damage response and alternative mechanisms. Cancers (Basel) 11: 1983, 2019.

36. Zhang W,LiQ, Song Cand Lao L: Knockdown of autophagy-related protein 6, Beclin-1, decreases cell growth, invasion, and metastasis and has a positive effect on chemotherapy-induced cytotoxicity in osteosarcoma cells. Tumour Biol 36: 2531-2539, 2015.

37. Xu R, Liu S, Chen H and Lao L: MicroRNA-30a downregulation contributes to chemoresistance of osteosarcoma cells through activating Beclin-1-mediated autophagy. Oncol Rep 35: 1757-1763, 2016.

38. Heras-Sandoval D, Pérez-Rojas JM, Hernández-Damián J and Pedraza-Chaverri J: The role of PI3K/AKT/mTOR pathway in the modulation of autophagy and the clearance of protein aggregates in neurodegeneration. Cell Signal 26: 2694-2701, 2014.

39. Li M, Hou Y, Wang J, Chen X, Shao ZM and Yin XM: Kinetics comparisons of mammalian Atg4 homologues indicate selective preferences toward diverse Atg8 substrates. J Biol Chem 286: 7327-7338, 2011

40. Shi M, Zhang T, Sun L, Luo Y, Liu DH, Xie ST, Song XY, Wang GF, Chen XL, Zhou BC and Zhang YZ: Calpain, Atg5 and Bak play important roles in the crosstalk between apoptosis and autophagy induced by influx of extracellular calcium. Apoptosis 18: 435-451, 2013.

41. Guo Y, Huang C, Li G, Chen T, Li J and Huang Z: Paxilitaxel induces apoptosis accompanied by protective autophagy in osteosarcoma cells through hypoxia-inducible factor-1 $\alpha$ pathway. Mol Med Rep 12: 3681-3687, 2015.

42. Peng B, Xu L, Cao F, Wei T, Yang C, Uzan G and Zhang D: HSP90 inhibitor, celastrol, arrests human monocytic leukemia cell U937 at G0/G1 in thiol-containing agents reversible way. Mol Cancer 9: 79, 2010

43. Kannaiyan R, Manu KA, Chen L, Li F, Rajendran P, Subramaniam A, Lam P, Kumar AP and Sethi G: Celastrol inhibits tumor cell proliferation and promotes apoptosis through the activation of c-Jun N-terminal kinase and suppression of PI3 K/Akt signaling pathways. Apoptosis 16: 1028-1041, 2011.
44. Sethi G, Ahn KS, Pandey MK and Aggarwal BB: Celastrol, a novel triterpene, potentiates TNF-induced apoptosis and suppresses invasion of tumor cells by inhibiting NF-kappaB-regulated gene products and TAK1-mediated NF-kappaB activation. Blood 109: 2727-2735, 2007.

45. Yang H, Chen D, Cui QC, Yuan X and Dou QP: Celastrol, a triterpene extracted from the Chinese 'Thunder of God Vine,' is a potent proteasome inhibitor and suppresses human prostate cancer growth in nude mice. Cancer Res 66: 4758-4765, 2006.

46. Hou W, Liu B and Xu H: Celastrol: Progresses in structure-modifications, structure-activity relationships, pharmacology and toxicology. Eur J Med Chem 189: 112081, 2020.

47. Beauchamp EM and Uren A: A new era for an ancient drug: Arsenic trioxide and Hedgehog signaling. Vitam Horm 88: 333-354, 2012.

48. Hashmi H and Nishihori T: Role of hematopoietic cell transplantation in relapsed acute promyelocytic leukemia. Clin Transplant 34: e14009, 2020.

49. Zhang G, Liu J, Zhang Y, Qu J, Xu L, Zheng H, Liu Y and Qu X: Cbl-b-dependent degradation of FLIP(L) is involved in ATO-induced autophagy in leukemic K562 and gastric cancer cells. FEBS Lett 586: 3104-3110, 2012

50. Jóźwiak S, Sadowski K, Kotulska K and Schwartz RA: Topical use of mammalian target of rapamycin (mTOR) inhibitors in tuberous sclerosis complex-A comprehensive review of the literature. Pediatr Neurol 61: 21-27, 2016.

51. Garza-Lombó C and Gonsebatt ME: Mammalian target of rapamycin: Its role in early neural development and in adult and aged brain function. Front Cell Neurosci 10: 157, 2016.

52. Pulakat L and Chen HH: Pro-senescence and anti-senescence mechanisms of cardiovascular aging: Cardiac MicroRNA regulation of longevity drug-induced autophagy. Front Pharmacol 11: $774,2020$.

53. Wang J, Li X, Zhong M, Wang Y, Zou L, Wang M, Gong X, Wang $X$, Zhou C, Ma X and Liu M: miR-301a suppression within fibroblasts limits the progression of fibrosis through the TSC1/mTOR pathway. Mol Ther Nucleic Acids 21: 217-228, 2020.

54. Cao L and Niu Y: Triple negative breast cancer: special histological types and emerging therapeutic methods. Cancer Biol Med 17: 293-306, 2020

55. Saraf AJ, Fenger JM and Roberts RD: Osteosarcoma: Accelerating progress makes for a hopeful future. Front Oncol 8: 4, 2018.

56. Kocaturk NM, Akkoc Y, Kig C, Bayraktar O, Gozuacik D and Kutlu O: Autophagy as a molecular target for cancer treatment. Eur J Pharm Sci 134: 116-137, 2019.

57. Ebrahimi S, Hosseini M, Shahidsales S, Maftouh M, Ferns GA, Ghayour-Mobarhan M, Hassanian SM and Avan A: Targeting the Akt/PI3K signaling pathway as a potential therapeutic strategy for the treatment of pancreatic cancer. Curr Med Chem 24: 1321-1331, 2017.

58. Bernardo PS, Lemos LGT, de Moraes GN and Maia RC Unraveling survivin expression in chronic myeloid leukemia: Molecular interactions and clinical implications. Blood Rev 43: $100671,2020$.

59. Waligórska-Stachura J, Jankowska A, Waśko R, Liebert W, Biczysko M, Czarnywojtek A, Baszko-Błaszyk D, Shimek V and Ruchała M: Survivin-prognostic tumor biomarker in human neoplasms-review. Ginekol Pol 83: 537-540, 2012.

60. Khan Z, Khan AA, Yadav H, Prasad GBKS and Bisen PS: Survivin, a molecular target for therapeutic interventions in squamous cell carcinoma. Cell Mol Biol Lett 22: 8, 2017.

61. Coumar MS, Tsai FY, Kanwar JR, Sarvagalla S and Cheung CH: Treat cancers by targeting survivin: Just a dream or future reality? Cancer Treat Rev 39: 802-811, 2013.

62. Church DN and Talbot DC: Survivin in solid tumors: Rationale for development of inhibitors. Curr Oncol Rep 14: 120-128, 2012.

63. Agostinis P, Berg K, Cengel KA, Foster TH, Girotti AW, Gollnick SO, Hahn SM, Hamblin MR, Juzeniene A, Kessel D, et al: Photodynamic therapy of cancer: An update. CA Cancer J Clin 61: 250-281, 2011.

64. Calabrò G, Patalano A, Lo Conte V and Chianese C: Photodynamic chemotherapy in the treatment of superficial mycoses: An evidence-based evaluation. G Ital Dermatol Venereol 148: 639-648, 2013

65. Carina V, Costa V, Sartori M, Bellavia D, De Luca A, Raimondi L, Fini M and Giavaresi G: Adjuvant biophysical therapies in osteosarcoma. Cancers (Basel) 11: 348, 2019. 